\title{
Associations Between Stair Climb Performance, Ultrasound Measured Muscle Thickness of the Lower Limbs and Self-Reported Pain and Function in Patients with Knee Osteoarthritis
}

Jenieve Guevarra' ${ }^{1}$, Rachel Welbel ${ }^{2}$ and Alfred C. Gellhorn ${ }^{3 *}$

*Correspondence: alg9109@med.cornell.edu

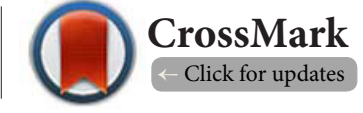

${ }^{1}$ Rutgers New Jersey Medical School - Newark, New Jersey.

2,3 Department of Rehabilitation Medicine, Department of Sports Medicine, Weill Cornell Medicine- New York City, NY.

\begin{abstract}
Background: Knee osteoarthritis (OA) commonly limits stair climbing. This study aimed to determine if self-reported pain and function and biomechanics during stair climbing were associated with ultrasound measured lower limb muscle thickness and isometric strength testing.

Methods: Twenty-three patients with unilateral symptomatic knee OA were recruited from a musculoskeletal physiatry clinic. Patients answered the WOMAC questionnaire and Lower Extremity Activity Scale to assess subjective pain, stiffness, function, and daily physical activity. Patients thenperformed a stair climb test. Ultrasound measurements of the quadriceps, hip abductors, adductors, and ankle plantarflexors were obtained. Isometric muscle strength was assessed with a hand-held dynamometer. Data analysis utilized Pearson correlations.

$\underline{\text { Results: }}$ Slower stair climb is associated with higher pain levels $(\mathrm{r}=0.75)$ and functional deficits $(\mathrm{r}=0.69)$. Ultrasound measured ankle plantarflexor thickness correlates with faster stair descent $(r=0.64)$. Stair climb ascent speed is negatively correlated with muscle strength of the hip adductors $(\mathrm{r}=-0.55)$ and abductors $(\mathrm{r}=-$ $0.55)$.

Conclusions: Stair climb speed is associated with self-reported pain and function. Ankle plantarflexors, hip adductor and abductor muscles should be explored as clinical targets for knee OA therapy.
\end{abstract}

Keywords: Knee osteoarthritis, ultrasound, WOMAC, stair climb

\section{Introduction}

Knee osteoarthritis (OA) is a prevalent joint disorder affecting $10 \%$ of men and $13 \%$ of women aged 60 years or older in the USA [1]. As such, knee OA is a primary cause of knee pain and functional limitation and can impede daily activities, including stair climbing [2]. In residences and facilities that cannot provide full elevator or escalator accessibility, the ability to independently ascend and descend stairs is a frequent daily necessity. Thus, clinicians treating patients with knee OA should be aware if the pain and functional deficits caused by knee OA are enough to impede stair climb ability. Pain, stiffness, and strength deficits in patients with knee OA can precipitate kinematic alterations in stair stepping such as increased plantar flexion during the swing phase of stair climbing and greater anterior pelvic tilt of the hips [3]. In addition to biomechanical compensations, knee OA patients' beliefs in their capabilities to execute actions have been shown to be crucial in physical task performance [4]. It is unknown whether self-rated assessments of pain, function, and lower extremity activity can predict how well knee OA patients can ascend and descend stairs. It is also unknown whether specific lower extremity muscle groups play a larger role than others in performing challenging functional 
motor tasks in this population. An ideal protocol to assess the functionality of specific muscle groups particularly in the realm of more demanding functional motor tasks such as the stair climb has yet to be established.

In addition to providing pharmacological or procedural treatment for pain and function, clinicians will also target specific lower extremity muscle weakness through exercise and physical therapy. Therefore, if the muscle bulk or muscle strength of certain lower extremity muscles have a greater impact on stair climb ability, these muscle groups should be identifiedin order to tailor treatment plans. Advanced crosssectional imaging tools such as CT and MRI can assess muscle bulk, but are impractical for clinical use. A minimally invasive and rapid tool that could be used in a clinical settingwould be ideal in orderto assess the functionality and strength of the muscle groups affected by knee OA. Ultrasound has emerged as a safe, cost-effective, and reliable method to measure muscle thickness that is typically readily accessible during a clinic visit. Recent studies have correlated ultrasound measured muscle thickness of the lower-limb muscles with standardized self-rated assessments of pain and function in patients with knee OA [5]. However, it is unknown whether ultrasound measured muscle thickness is associated with performance in functional motor tasks such as the stair climb.

The primary aim of this study is to determine if performance on an office based stair climbing functional test is associated with thickness of specific muscle groups measured by ultrasound. The secondary aim of this study is to determine if self-rated pain, function, and lower extremity activity are associated with stair climb ability. Although there is published literature reporting the kinematic and biomechanical modifications that occur in knee OA patients while climbing stairsthere have been no assessments of how these chronic accommodations are reflected in the strength and measurement of specific muscle groups, to our knowledge. Given the varying level of strength, proprioception, and coordination in different muscles required in stair ascent in comparison to stair descent [6], we theorize that our analysis will reveal ultrasound measured muscle thickness of specific muscle groups activated during stair ascent will correlate with faster stair ascent speed. We predict analogous findings for muscles activated during stair descent in relation to stair descent speed. This finding would suggest that the protocol of measuring muscle thickness using ultrasound can be used to target specific muscle groups for physical therapy in patients with knee OA.

Furthermore, we hypothesize that increased self-rated pain, functionaldeficits and decreased lower extremity activitywill be associated with longer stair climb times.By evaluating selfrated assessments of pain, function and activity, we are also able to assess the accuracy of a patient's perception of stair climbing limitations and possible compensations.

\section{Materials and methods}

Subjects were recruited from a musculoskeletal physiatry clinic at an academic medical center. To meet inclusion criteria for the study subjects met at least 3 of the following: at least 50 years old, presence of knee stiffness that lasts for at least 30 minutes in the morning, presence of grating or crunching sounds in the knee, tenderness to palpation of the bony prominences around the knee, presence of bony enlargement around the knee, and the absence of warmth to touch on the knee. They also were required to have $>3 / 10$ pain when walking and a prior x-ray of the knee with evidence of OA.

Subjects were excluded from the study if they had any of the following: pain predominantly on the lateral aspect of the knee, a prior corticosteroid injection into the knee within 4 weeks, and a prior diagnosis of a neuromuscular condition that affected lower extremity strength. In addition, the subjects were also asked if they had contralateral knee pain $>3 / 10$.

All subjects were screened either in person or via telephone call and met inclusion criteria before being enrolled in the study.Informed consent forms which explained information regarding procedures, the possible benefits and risks of participation, how to later acquire results of the research, option of withdrawing, and the contact information of the research coordinators were provided. Informed consent was obtained by a research coordinator in-person before beginning the study. Subjects were asked to fill out three additional questionnaires. The first questionnaire provided the researchers with general characteristics (weight, height, symptomatic side) of the subjects and a subjective scale of current pain from 0 to 10 , with 10 being more severe pain. The Western Ontario and McMaster Universities Arthritis Index (WOMAC) was used to assess subjects' pain, stiffness, and physical functioning based on subjective answers on a scale from none to extreme for 24 inquiries. The Lower Extremity Activity Scale (LEAS) was used to determine the level of daily physical activity in each patient. Subjects chose the closest description of their activity level from 18 possible options.Local Institutional Review Board approval was obtained.

\section{Stair Climb}

Stair climb power was measured in a method similar to Bean et al [7]. The examiner directed the subject to the designated stairway located in the clinic and the subjects were instructed to stand a comfortable distance away from the first step. If desired, they were allowed to use the handrail on the preferred side for safety. A stopwatch was started when the subject began to step and was stopped when the subject reached the top of the staircase with both feet. They were instructed to ascend the stairs at a comfortable pace and were able to stop if at any time they felt unsafe. Subjects were then instructed to descend the stairs and the stopwatch was started and stopped analogously to ascent. Stair climb time here is defined as the total amount of time the subject took to ascend and descend a full flight of stairs. To calculate stair climb power, power was calculated as force times velocity. Force was calculated using the body mass of the subject in kg times gravitational acceleration. Velocity was calcu- 
Guevarra et al, Physical Therapy and Rehabilitation 2019,

lated using stair height and divided bystair climb ascent time.

\section{Ultrasound Measurements}

The following muscle groups were measured under ultrasound: quadriceps, hip abductors, hip adductors, and plantar-flexors. A mark was placed at half of the distance between the greater trochanter and the lateral condyle of the femur identified by palpation. This line was extended across the anterior and medial leg to obtain consistent imaging of the quadriceps and adductors. Next, the distance between the lateral femoral condyle and the lateral malleolus was measured and recorded. A mark was placed at $1 / 3$ of this measurement distally from the lateral condyle for consistent US imaging of the gastrocnemius and soleus. A final measurement was taken from the anterior superior iliac spine to the greater trochanter of the femur, and a mark was placed at half of this distance. This measurement was used for US imaging of the gluteus medius and minimus (hip abductors).

For US imaging, the subject was asked to lie relaxed in a supine position on an examining table. ASonoSite X-Porte (Bothell, WA) ultrasound machine and a curvilinear transducer (5-1 MHz) were used to obtain all images. The transducer was placed in the transverse plane to achieve the most accurate image possible. After the muscle was identified, the examiner slightly retracted the probe as to not compress the muscle. This static image was captured. The examiner measured maximum muscle thickness using the measuring tools available on the US machine. The image was saved and the process was repeated three times for each muscle group (quadriceps, hip abductors, hip adductors, and ankle plantarflexors). All three measurements were recorded. Once all images were obtained from one lower extremity, the same method was used for imaging of the other. Ratios of knee extensors to hip abductors and ratios of knee extensors to ankle plantar flexors were calculated to compare the relative thickness of muscles highly activated during ascent (hip abductors) versus the relative thickness of muscles highly activated during descent (ankle plantarflexors).

\section{Strength Measurements}

After US measurements were completed, strength testing was conducted. A hand-held dynamometer (HHD) was used to measure peak force over a 3 second period, as per settings on the dynamometer. Anatomical markers were used for dynamometer placement to achieve accurate lever arm measurements. When obtaining measurements for the hip abductors, the HHD was placed $5 \mathrm{~cm}$ proximal to the lateral malleolus. For the adductors, the HHD was placed $5 \mathrm{~cm}$ proximal to the medial malleolus. For the quadriceps, the HHD was placed midline at $5 \mathrm{~cm}$ proximal to the lateral malleolus. Finally, for the plantarflexors, the HHD was placed at the metatarsophalangeal joint centered between the first and second digits.
All of our tests were "make tests" such that the subject pushed against the HHD while the examiner maintained the HHD as steady and as consistently placed as possible. For each test, the subject was allowed to have one warm-up ( $~ 50 \%$ maximum strength) to account for any habituation. The test was repeated three times for each muscle group. The subjects were given a 30 second rest period between each of the tests performed so as not to fatigue them. All tests lasted 3 seconds as determined by the dynamometer. The settings on the machine itself were set to stop recording with an audible beep after this time period had elapsed. To initiate each test, the subjects were instructed to "go" then the examiner repeated "push, push, push" to signal the patient to push as hard as possible for the remaining three seconds of the test. After the HHD beeped, the examiner told the subject to "relax" as to signal the end of the test. Maximal force attained during each attempt was recorded. Similar to ultrasound measured muscle thickness, ratios of knee extensors to hip abductors and ratios of knee extensors to ankle plantar flexors were calculated to compare the relative strength of muscles highly activated during ascent (hip abductors) versus the relative strength of muscles highly activated during descent (ankle plantar flexors).

\section{Data analysis}

All statistical analysis was performed using STATA 14.1 (College Station, TX), with alpha level for hypothesis testing set at 0.05 . We used simple descriptive statistics to describe our cohort, and paired t-tests to evaluate for any differences in muscle parameters between symptomatic and asymptomatic limbs. We used Pearson correlation coefficients to evaluate the relationship between baseline characteristics and muscle measures, as well as between functional measures and muscle parameters. We considered $r$ values $<0.3$ to represent a weak association, $0.3-0.7$ to represent a moderate association, and $>0.7$ to represent a strong association.

\section{Results}

Subjects in this study included 23 adults with unilateral symptomatic knee osteoarthritis. The subjects included 12 females and 11 males with the average age of $63.8 \pm 9.3$ years and median symptom duration of knee OA of 2 years. 12 subjects had right-sided symptomatic knee OA while 11 had left-sided knee OA. The baseline characteristics of the subjects used in this study are shown in Table 1. Symptoms as reported by the WOMAC index were mild to moderate with a mean total score of 25 on a scale from 0 to 96 where higher values indicate worse symptoms. LEAS scores measured self-reported functional daily activity and had a mean score of 13.1 on a scale of 1-18, where higher values were indicative of higher daily functional activity. The ultrasound measured muscle bulk measurements and strength measurements of the subjects showed no statistically significant differences between symptomatic and asymptomatic limbs. 
Guevarra et al, Physical Therapy and Rehabilitation 2019,

Table 1. Subject Baseline Characteristics, N=22.

\begin{tabular}{|c|c|c|}
\hline & & Mean (SD) or percent \\
\hline Age & & $63.8(9.3)$ \\
\hline Gender (Female) & & $52 \%$ \\
\hline Weight (kg) & & $77.4(14.5)$ \\
\hline BMI & & $26.9(3.7)$ \\
\hline Pain Level & & $4.1(1.8)$ \\
\hline Symptomatic side (Right) & & $52 \%$ \\
\hline Symptom Duration (Months) & & $44.8(62.1)$ \\
\hline \multicolumn{3}{|l|}{ Symptomatic KL Grade } \\
\hline & 0 & 0 \\
\hline & 1 & 1 \\
\hline & 2 & 9 \\
\hline & 3 & 12 \\
\hline & 4 & 0 \\
\hline \multicolumn{3}{|l|}{ Asymptomatic KL Grade } \\
\hline & 0 & 8 \\
\hline & 1 & 7 \\
\hline & 2 & 7 \\
\hline & 3 & 0 \\
\hline & 4 & 0 \\
\hline WOMAC pain subscale $(0-20)$ & & $4.6(3.2)$ \\
\hline WOMAC stiffness subscale $(0-8)$ & & $3.1(1.7)$ \\
\hline WOMAC function subscale (0-68) & & $17.45(13.3)$ \\
\hline WOMAC total (0-96) & & $25.3(17.4)$ \\
\hline LEAS Score $(0-18)$ & & $13.09(2.8)$ \\
\hline
\end{tabular}

NRS: Numeric Rating System

BMI: Body Mass Index

KL: Kellgren Lawrence System

WOMAC: Western Ontario and McMaster Arthritis Index

LEAS: Lower Extremity Activity Scale

Correlations between stair climb test performance and functional measures

Table 2 reports unadjusted correlations between self-reported functional measures (WOMAC and LEAS scales) and perfor- mance on the stair climb functional test. Slower total stair climb speed was strongly correlated with increased WOMAC pain ratings $(r=0.75)$. The strongest correlation in the study was between the WOMAC function subscale and stair ascent $(r=0.82)$.Notably, slower stair ascent wasalso strongly associated with increased WOMAC pain subscale $(r=0.76)$ and WOMAC total score $(r=0.80)$. and moderately related tolower extremity activity. In terms of descent, slower stair climb descent showed moderate associations with increased WOMAC pain and function ratings, total WOMAC scores and improved lower extremity activity ratings.

Correlations between stair climb test performance and ultrasound measured muscle thickness

Table 3 details unadjusted correlations between stair climb test performance and ultrasound muscle thickness of the symptomatic knee normalized to subject weight. Slower total stair climb speed showed moderate associations with ultrasound measured muscle thickness of the quadriceps $(r=-0.51)$, hip adductors $(r=-0.52)$, and ankle plantar flexors $(r=-0.64)$. Notably, the increased thickness of the ankle plantar flexors also had the strongest correlation with faster stair ascent of all muscle groups $(r=0.54)$.

\section{Correlations between stair climb test performance muscle strength}

Table 3 describes the unadjusted correlations between stair climb test performance and muscle strength measured as torque of the symptomatic knee normalized to subject weight. Decreased stair climb speed was moderately associated with diminished muscle strength of the hip abductors, hip adductors, and ankle plantar flexors. The strongest correlation in all comparisons of stair climb performance and muscle strength was seen between hip adductor strength and increased stair climb power $(r=0.71)$. On separate analysis of ascent and descent, a slower stair climb ascent was associated with a decreased muscle strength in the hip abductors, hip adductors, and ankle plantar flexors. There were no significant associa-

Table 2. Unadjusted Pearson correlation between stair climb test performance and functional measures.

\begin{tabular}{llllll}
\hline & $\begin{array}{l}\text { WOMAC Pain } \\
\text { Subscale }\end{array}$ & $\begin{array}{l}\text { WOMAC } \\
\text { Stiffness } \\
\text { Subscale }\end{array}$ & $\begin{array}{l}\text { WOMAC } \\
\text { Function } \\
\text { Subscale }\end{array}$ & $\begin{array}{l}\text { WOMAC } \\
\text { Total }\end{array}$ & LEAS \\
\hline Stair Climb Time (seconds) & $\mathbf{0 . 7 5 ^ { * }}$ & 0.21 & $0.69^{*}$ & $0.69^{\star}$ & $-0.55^{*}$ \\
Stair Climb Power (Ascent) (Watts) & $-0.62^{*}$ & -0.31 & $-0.53^{*}$ & $-0.55^{*}$ & 0.28 \\
Stair Climb Ascent Time(seconds) & $\mathbf{0 . 7 6 ^ { * }}$ & 0.36 & $\mathbf{0 . 8 2}^{*}$ & $\mathbf{0 . 8 0}^{*}$ & $-0.44^{*}$ \\
Stair Climb Descent Time (seconds) & $0.66^{*}$ & 0.10 & $0.54^{*}$ & $0.55^{*}$ & $-0.54^{*}$ \\
Ratio of Ascending to Descending Time & -0.38 & -0.03 & -0.19 & -0.22 & $0.47^{*}$ \\
\hline
\end{tabular}

LEAS: Lower Extremity Activity Scale

WOMAC: Western Ontario and McMaster Arthritis Index

Values indicated by ${ }^{\star}$ with indicates significance at 0.05 level.

Values bolded indicate a strong correlation $(r>0.7)$ 
Guevarra et al, Physical Therapy and Rehabilitation 2019,

http://www.hoajonline.com/journals/pdf/2055-2386-6-4.pdf

doi: $10.7243 / 2055-2386-6-4$

Table 3. Unadjusted correlation between stair climb test performance and ultrasound muscle thickness measures (mm) normalized to weight $(\mathrm{kg})$ of the Symptomatic Knee.

\begin{tabular}{|c|c|c|c|c|c|c|}
\hline & $\begin{array}{l}\text { Knee Extensors } \\
\text { (Quadriceps) }\end{array}$ & Hip Abductors & Hip Adductors & $\begin{array}{l}\text { Ankle plantar } \\
\text { flexors }\end{array}$ & $\begin{array}{l}\text { Ratio of Knee } \\
\text { Extensors to Hip } \\
\text { Abductors }\end{array}$ & $\begin{array}{l}\text { Ratio of Knee } \\
\text { Extensors to Ankle } \\
\text { Plantar Flexors }\end{array}$ \\
\hline Stair Climb Time (seconds) & $-0.51^{\star}$ & -0.39 & $-0.52^{*}$ & $-0.64^{*}$ & -0.07 & $0.61^{*}$ \\
\hline Stair Climb Power (Watts) & 0.16 & 0.01 & $<0.01$ & 0.12 & 0.12 & -0.21 \\
\hline $\begin{array}{l}\text { Stair Climb Ascent Time } \\
\text { (seconds) }\end{array}$ & $-0.52^{\star}$ & -0.29 & $-0.45^{*}$ & $-0.54^{*}$ & -0.18 & $0.47^{\star}$ \\
\hline $\begin{array}{l}\text { Stair Climb Descent Time } \\
\text { (seconds) }\end{array}$ & $-0.45^{*}$ & -0.40 & $-0.49^{*}$ & $-0.62^{*}$ & -0.01 & $0.62^{\star}$ \\
\hline $\begin{array}{l}\text { Ratio of Ascending to } \\
\text { Descending Time }\end{array}$ & 0.25 & 0.25 & 0.31 & 0.39 & 0.03 & -0.37 \\
\hline
\end{tabular}

Values indicated by ${ }^{*}$ with indicates significance at 0.05 level.

Values bolded indicate at least moderate correlations $(r>0.5)$

tions between stair climb descent time and muscle strength.

\section{Discussion}

The main findings of this study were the correlations revealed between self-rated pain and function, as reported through the WOMAC and LEAS, and stair climb performance. In addition, this study elucidated associations between US measured muscle thickness and isometric strength of specific lower extremity muscle groups and stair climb ability.

\section{Self-Reported Indices and Stair Climb Performance}

This study reported strong correlations between self-rated pain and function, as reported through the WOMAC index, and stair climb performance. Namely, the highest associations of the entire analysis were seen between stair climb ascent time and the WOMAC pain and function subscales, and WOMAC total score. Similar strong correlations were found between increased self-reported pain on the WOMAC pain subscale and prolonged total stair climb. However, given the high correlation between the subject WOMAC pain and function indices and stair ascent, it is likely that the prolonged stair ascent time was the main contributing factor to the increased total stair climb time.

Stair ascent requires lower extremity muscles to generate enough force to carry the body against gravity, making stair ascent, even in healthy individuals, more energetically challenging than descent [8]. Patients with knee OA tend to exhibit biomechanical negotiations such as increased hip abduction and decreased knee flexion on stair climb [9]. These kinematic changes, which decrease stair stepping efficiency, may be the cause of the observed longer ascent times that correlate with increased knee pain. Another consideration for the slower stair ascent is whether pain avoidance or kinesiophobia (fear that movement can cause further injury) can affect stair climb ability. Prior studies utilized the Tampa Scale for Kinesiophobia in patients with knee OA pre-and post-knee replacement surgery and found a strong relationship between the fear of falling and stair climb ability [10].

In addition to stair climb time, stair climb power, as measured in watts, showed a strong negative correlation with WOMAC Pain subscale ratings indicating thatwith decreased pain, more power was exerted during the duration of the total stair climb. Compared to isometric strength, stair climb power takes into account the speed of movement and accelerations of the body's center of mass, allowing it to be a reliable predictor of outcomes of mobility performance [7]. In addition, decreased reported pain signifies a reduction in the protective pain withdrawal reflex which would allow patients to place increased joint loads on the knee [11]. Coupled with the results of this study, it is reasonable to conclude that reduced subjective pain allows patients to increase weight bearing on the knee joint which will allow for the application of boosted leg muscle power during stair climb.

The correlations between self-rated assessments of pain and function with stair climb power and stair climb time imply that the ability to climb stairs involves an interplay of biomechanical accommodation, pain perception, and lower extremity mobility. Future studies would aim to further explore the relationship between subjective pain and function to delineate the degree to which the effects seen during stair climbing in patients with knee OA is influenced by kinematic negotiations, the inability to fully load the muscles, and pain cognizance.

\section{Ultrasound Measured Muscle Thickness and Stair Climb Performance}

Given recent studies purporting that ultrasound measured muscle thickness is correlated with pain and function, it was theorized that the thickness of distinct lower extremity muscle groups involved in stair ascent and/or descent would also be connected with stair climbing ability. In assessing the possible associations between stair climb test performance, lower extremity activity, and ultrasound measured muscle thickness, the highest correlation values were seen between the muscle thicknesses of the ankle plantar flexors and stair 
climb test performance. Specifically, as ankle plantar flexor thickness increases, total stair climb speed increases.

Previous literature describes the stages of stair descent as consisting of a stance phase made up of weight acceptance, forward continuance, and controlled lowering and a swing phase consisting of leg pull through and preparation of foot placement. During the controlled lowering portion of the stance phase, the knee extensors and ankle plantar flexors undergo eccentric contraction in order to prepare to absorb energy for foot placement on the descending stair [12]. The role of ankle plantar flexors in absorbing force has been studied in the knee OA population in published studies on gait. In particular, ankle plantar flexors have been found to predict compressive knee joint reaction force. Specifically, ankle plantar flexorscan restrain forward rotation of the tibia about the ankle in turn modifying joint reaction force on the femur. Patients with knee OA will adjust their gait by decreasing ankle plantar flexion and increasing ankle dorsiflexion thus reducing gait velocity. These compensations slow the rate of loading onto the knee joint as well as allow the tibia to rotate forward, effectively decreasing compressive knee joint reaction force [13]. Taken with the findings of this study, these data provide strong evidence that the negotiations occurring during level walking likely occur on stair descent. It is possible that patients with knee OA with slower stair descent speed maydecrease ankle plantar flexion to slow joint loading. At this time, this study cannot determine causality between biomechanical negotiations and changes muscle thickness. Yet, the results do imply that ankle plantar flexors are associated with faster stair descent and overall stair climb speed.

\section{Muscle Strength as Torque and Stair Climb Performance} The strongest correlation between muscle strength measured as torque and stair climb performance was seen between hip adductors and stair climb power. Our findings indicate that increases in measured hip adductor strength has a moderate correlation with increased stair climb power. There was also a slightly weaker moderate correlation seen in hip abductors. Increases in hip abductor and adductor strength were moderately associated with faster stair ascent speed. Previous literature characterizes the stages of stair ascent as a stance phase consisting of weight acceptance, pull up, forward continuance and a swing phase consisting of foot clearance and foot placement [12]. During stair ascent, the hip abductors on the ascending limb activate in order to laterally rotate the trunk over the stance limb. The hip adductors play less of a clear role during stair climb. In general, hip adductors help accurately position the lower limb during gait; they likely play a similar role during stair climb. Given that patients with knee OA may report increased amounts of knee instability, we theorize that patients who are able to quickly ascend the stairs have compensated through increased hip adductor strength. Futurebiomechanical studies should target this muscle group to better define its role in stair climbing in patients with knee OA.

\section{Ultrasound measured muscle thickness versus Isometric Muscle Strength}

Surprisingly, the strongest correlation between US measurement thickness of the plantarflexors and stair descent time was not replicated when looking at isometric muscle strength. This may be related to the difficulty of using a hand-held dynamometer to accurately assess plantarflexor strength. Because of the high torque generated by the ankle plantarflexors, this muscle group can overcome the strength of the examiner when manually testing the muscle. Because of this, ultrasound is especially attractive as a method to assess the ability to generate ankle plantarflexor force. The usage of ultrasound to measure muscle bulk provides measurement that would not vary with tester strength. Ultrasound is not without its limitations, and measurements of the hip and thigh muscles may be more challenging due to increased subcutaneous tissue, less often seen in the lower legs, and may also be why stronger correlations were not seen between these muscles with stair climb activity. These differences suggest that specific muscle characteristics must be taken into account when deciding on the method of analysis.

\section{Limitations}

This study had important limitations. The relatively small sample size impeded a more powerful analysis such as regression analyses. Furthermore, the cross-sectional design of this study hinders any conclusions about the causality of the relationships revealed here. As mentioned, the ultrasound measurements and isometric muscle strength measurements have inherent limitations due to variations in strength of the examiners relative to the subjects and body composition of the subjects. Although a regression analysis would have provided more robust evidence of the role of each muscle as a predictor of stair climb performance, the clear evidence of stronger correlations between the strength and bulk of distinct muscle groups, self-reported pain, function, and mobility indices, and stair climb ability imply that differential negotiations occur in the muscles of the symptomatic limb in patients with knee OA.

\section{Conclusion}

This study found that self-reported ratings of pain and function strongly correlate with the ability to climb stairs. Additionally, muscle bulk of the ankle plantar flexors as measured with ultrasound was associated with faster stair descent. Finally, muscle strength of the hip adductor and hip abductor muscles was correlated with faster stair descent ascent. Simple office-based tests, such as ultrasound measurements of specific muscle groups, most notably, the plantar flexors, and the WOMAC questionnaire may be a strong indicator of stair climb function. In patients with decreased stair climb mobility, either noted from self-reported surveys, stair climb 
Guevarra et al, Physical Therapy and Rehabilitation 2019,

functional testing, ultrasound measurements or dynamometer testing, treatments should target increasing muscle strength in the ankle plantarflexors, hip adductors and hip abductors.

\section{Competing interests}

The authors declare that they have no competing interests.

Authors' contributions

\begin{tabular}{|l|c|c|c|}
\hline Authors' contributions & JG & RW & ACG \\
\hline Research concept and design & $\checkmark$ & $\checkmark$ & $\checkmark$ \\
\hline Collection and/or assembly of data & $\checkmark$ & -- & $\checkmark$ \\
\hline Data analysis and interpretation & $\checkmark$ & -- & $\checkmark$ \\
\hline Writing the article & $\checkmark$ & $\checkmark$ & $\checkmark$ \\
\hline Critical revision of the article & $\checkmark$ & -- & $\checkmark$ \\
\hline Final approval of article & $\checkmark$ & $\checkmark$ & $\checkmark$ \\
\hline Statistical analysis & -- & -- & $\checkmark$ \\
\hline
\end{tabular}

Publication history

Editor: Mohammad H. Hadadzadeh, Wheeling Jesuit University, USA. Received: 05-Dec-2018 Final Revised: 10-Feb-2019

Accepted: 12-Feb-2019 Published: 03-Apr-2019

\section{References}

1. Zhang Y and Jordan JM. Epidemiology of osteoarthritis. Clin Geriatr Med. 2010; 26:355-69. | Article | PubMed Abstract | PubMed FullText

2. Whitchelo T, McClelland JA and Webster KE. Factors associated with stair climbing ability in patients with knee osteoarthritis and knee arthroplasty: a systematic review. Disabil Rehabil. 2014; 36:1051-60. | Article | PubMed

3. Goncalves GH, Selistre LF, Petrella M and Mattiello SM. Kinematic alterations of the lower limbs and pelvis during an ascending stairs task are associated with the degree of knee osteoarthritis severity. Knee. 2017; 24:295-304. | Article | PubMed

4. Maly MR, Costigan PA and Olney SJ. Contribution of psychosocial and mechanical variables to physical performance measures in knee osteoarthritis. Phys Ther. 2005; 85:1318-28. | PubMed

5. Gellhorn AC, Stumph JM, Zikry HE, Creelman CA and Welbel R. Ultrasound measures of muscle thickness may be superior to strength testing in adults with knee osteoarthritis: a cross-sectional study. BMC Musculoskelet Disord. 2018; 19:350. | Article | PubMed Abstract | PubMed FullText

6. Hicks-Little CA, Peindl RD, Fehring TK, Odum SM, Hubbard TJ and Cordova $\mathrm{ML}$. Temporal-spatial gait adaptations during stair ascent and descent in patients with knee osteoarthritis. J Arthroplasty. 2012; 27:1183-9. | Article I PubMed

7. Bean JF, Kiely DK, LaRose S, Alian J and Frontera WR. Is stair climb power a clinically relevant measure of leg power impairments in at-risk older adults? Arch Phys Med Rehabil. 2007; 88:604-9. | Article | PubMed

8. Brand RA, Pedersen DR and Friederich JA. The sensitivity of muscle force predictions to changes in physiologic cross-sectional area. J Biomech. 1986; 19:589-96. | Article | PubMed

9. Hicks-Little CA, Peindl RD, Hubbard TJ, Scannell BP, Springer BD, Odum SM, Fehring TK and Cordova ML. Lower extremity joint kinematics during stair climbing in knee osteoarthritis. Med Sci Sports Exerc. 2011; 43:516-24. | Article | PubMed

10. Unver B, Ertekin $O$ and Karatosun V. Pain, fear of falling and stair climbing ability in patients with knee osteoarthritis before and after knee replacement: 6 month follow-up study. J Back Musculoskelet Rehabil. 2014; 27:77-84. | Article | PubMed

11. Hurwitz DE, Ryals AR, Block JA, Sharma L, Schnitzer TJ and Andriacchi TP. Knee pain and joint loading in subjects with osteoarthritis of the knee. $J$
Orthop Res. 2000; 18:572-9. | Article | PubMed

12. McFadyen BJ and Winter DA. An integrated biomechanical analysis of normal stair ascent and descent. J Biomech. 1988; 21:733-44. | Article | PubMed

13. Robon MJ, Perell KL, Fang $M$ and Guererro E. The relationship between ankle plantar flexor muscle moments and knee compressive forces in subjects with and without pain. Clin Biomech (Bristol, Avon). 2000; 15:522-7. | Article | PubMed

\section{Citation:}

Guevarra J, Welbel R and Gellhorn AC. Associations Between Stair Climb Performance, Ultrasound Measured Muscle Thickness of the Lower Limbs and Self-Reported Pain and Function in Patients with Knee Osteoarthritis. Phys Ther Rehabil. 2019; 6:4. http://dx.doi.org/10.7243/2055-2386-6-4 